\title{
A Singular Perturbation Approach to Control of Lightweight Flexible Manipulators
}

\section{Bruno Siciliano}

Universita' di Napoli

Dipartimento di Informatica e Sistemistica

Via Claudio 21, 80125 Napoli, Italy

\section{Wayne J. Book}

Georgia Institute of Technology

G.W. Woodruff School of Mechanical Engineering

At Tanta, Georgia 30332

\section{Abstract}

The control of 1 ightweight flexible manipulators moving along predefined paths is the focus of this work. The flexible manipulator dynamics is derived on the basis of a Lagrangian-assumed modes method. The ful1 order flexible dynamic system does not a 110 the determination of a tracking control as for rigid manipulators, since there are not as many control inputs as output variables. This drawback is overcome by accomplishing a model order reduction, based on a singular perturbation strategy, where the perturbation parameter can be identified as the ratio of speeds of the slow versus the fast dynamics.

A composite control is adopted. First a slow control is designed with the purpose to track the trajectory in the joint space as accurately as possible. A quasi steady-state trajectory is then determined for the fast variables, and a fast control is in charge of stabilizing them along that trajectory.

The one link flexible arm prototype in the Flexible Automation Laboratory at Georgia Tech is chosen for developing a case study. Extensive simulation results are illustrated.

This research is based in part on work supported by the NATO Science Programme (Special Programme on Sensory Systems for Robotic Control) under grant 9.11.04 no. 586/85, and by the Computer Integrated Manufacturing Systems Program at Georgia Tech. 


\section{Introduction}

State-of-the-art design of mechanical arms results in a high ratio of arm weight to payload weight. The use of lightweight arms certainly represents one successful strategy to achieve benefits like lower arm cost, higher motion speeds, better energy efficiency, safer operation and improved mobility. These issues and others have been addressed by Book (1984 (August)).

In this scenario control is one of the crucial points to an effective use of Tighter arms. As a matter of fact the price to pay is the much more complex dynamics involved by the flexibility distributed along a lightweight mechanical structure. Consequentiy the control objective for flexible manipulators is to be properly reformulated, at least as compared to the case of today's industrial manipulators, whose links and drives are purposely made stiff to obtain higher precision, even if at the expenses of lower speed and higher drive power. In fact not on $1 y$ is a flexible manipulator required to execute some motion, so as a rigid manipulator is, but also it is desired to stabilize the vibrations, which are naturally excited, along the motion and damp them out as fast as possible at the terminal point. The dynamic interaction existing between joint angles and flexible deflections complicates the control problem so that alternative control strategies must be sought to accomp 7 ish the above mentioned goal.

The necessary step towards successful control synthesis is to dispose of an accurate dynamic model for the flexible manipulator; to this end the recursive Lagrangian-assumed modes method proposed by Book (1984) is an efficient, complete and conceptually straightforward modeling technique. The result is an extended number of generalized coordinates, and then state variables, to handle for control purposes.

If the control objective is just to drive the manipulator to a terminal 
point (regulator problem) and stabilize the deflections, without any concern about the particular path to follow, an optimal control approach is candidate to succeed, as proven for instance in (Sangveraphunsiri 1984) for a single link flexible arm.

On the other hand if the path followed is also of interest, this creates a conflict in some way with the intrinsically flexible nature of the manipulator. As a natural extension of the regulator problem, it might be argued that the nonlinear equations of motion could be linearized about the desired nominal rigid body motion, but this would involve much too an expensive computational burden. Furthermore, since the manipulator is likely required to handle a wide variety of payloads along trajectories avoiding obstacles in the workspace, robustness of the control system would be serious ly compromised.

A first research effort towards the design of a control law which decouples the joint angle motion from the flexible motion has been produced by Singh and Schy in (1985) but the resulting flexible motion stabilizer takes advantage of additional erid forces. A conceptually similar idea is the reaction whee 1 placed at the tip of a one link flexible arm operating in a space environment (Meldrum 1985). Ultimately also in case of the bracing arm, recently proposed by Book (Book and Sangveraphunsiri 1984), the sma 11 arm, which is in charge of the fine motion, could produce end stabilizing forces as regards the gross motion performed by the big flexible arm. Nevertheless, whenever only as many control inputs as joint variables are available, nonlinear control of combined rigid body and flexible motion seems to remain an unresolved issue.

The goal of this paper is to present a fairly different approach to the control of flexible manipulators moving along predefined paths, based on singular perturbation theory. A conceptually similar approach has been pursued 
by Marino and Nicosia (1985) for the simplified case of flexibility concentrated at joints. If the spectrum associated with the flexible modes characterizing the deflections is distant enough from the spectrum of the rigid body motion, the system can be considered a singularly perturbed one; the perturbation parameter can be identified as the ratio of speeds of the slow vs the fast dynamics.

According to the singular perturbation theory (see Kokotovic's comprehensive survey (1984)) a system in singular perturbation form is a convenient tool for "reduced order modeling". In this way two reduced order systems are identified: a slow subsystem which nicely turns out to be of the same order as that of a rigid manipulator, and a fast subsystem in which the slow state variables play the role of parameters. By following the composite control technique proposed by Chow and Kokotovic (1978), and later by Suzuki (1981), a slow feedback tracking control is designed first, then a fast feedback control is added, whose purpose is to stabilize the fast (boundary layer) subsystem along its equilibrium trajectory which obvious ly depends a iso on the slow control.

The attractive feature of this strategy is that the slow control can be designed on the basis of well established control schemes for rigid manipulators, such as the decoupling control proposed by Freund (1982), the resolved acceleration control by Luh, Walker and Paul (1980), the sliding mode control by Slotine (1985), and the adaptive model following control by Balestrino, De Maria and Sciavicco (1983). On the other hand the fast control can be synthesized as a linear state feedback control with the slow state variables acting as parameters; an optimal control can be conceived. In fact if the fast subsystem is stabilizable for any joint trajectory of interest, Tikhonov's theorem (Kokotovic 1984) will assure that the orbits of the overall 
system will approach in the 1 imit those derived by the two subsystems.

Preliminary satisfactory results with a two stage control strategy, based on mode 1 reference adaptive control for the full order system, have been obtained in (Siciliano et a 1. 1986) for the one link flexible arm prototype which has been focus of research at Georgia Tech. See (Hastings and Book 1986) for the dynamic mode 1. The same arm is chosen in this work to develop a case study, and simulation results are provided. However the application of the control technique out 1 ined in this paper to the general case of a multiple degree of freedom manipulator with one or more flexible links, like the case considered in (Singh and Schy 1985), appears much more challenging and it will soon be investigated.

Last but not least it must be mentioned that full state availability is assumed for control synthesis. As a matter of fact the flexible variables can be obtained from strain gage measurements as shown in (Hastings and Book 1985), whereas their derivatives can be derived through a reconstructing filter as in (Sangveraphunsiri 1984).

\section{Flexible Manipulator Dynamics}

Nonlinear equations of motion for a flexible manipulator can be successfully derived using the recursive Lagrangian approach out Tined by Book (1984a). A solution to the flexible motion of a link $i$ is obtained through modal analysis, under the assumption of small deflection of the link,

$$
\mathbf{u}_{i}(\eta, t)=\sum_{j=1}^{m_{i}} \delta_{i j}(t) \phi_{i j}(\eta)
$$

where $\phi_{i j}$ is the eigenvector expressing the displacement of mode $j$ of 1 ink $i$ 's deflection, $\delta_{i j}$ is the time-varying amplitude of mode $j$ of 1 ink $i$, and $m_{i}$ is 
the number of modes used to describe the deflection of 1 ink $i$. As far as the external forcing terms in Lagrange's formulation, if the clamped-free assumption is adopted for a flexible link, there will be no displacements at joint locations and then the corresponding generalized forces will be zero. Thus, similarly to the Maizza Neto's formulation (1974), for the purpose of this work, the dynamic equations for an $n$-degree-of-freedom manipulator with up to $n$ flexible links can be written in the following form:

$$
\mathbf{M}(\mathbf{q}, \delta)\left[\begin{array}{c}
\ddot{\mathrm{q}} \\
\hdashline \ddot{\delta}
\end{array}\right]+\left[\begin{array}{c}
\mathrm{f}_{1}(\mathrm{q}, \dot{\mathrm{q}}) \\
\hdashline \mathbf{f}_{2}(\mathbf{q}, \dot{\mathrm{q}})
\end{array}\right]+\left[\begin{array}{c}
\mathrm{c}_{1}(\mathbf{q}, \dot{\mathbf{q}}, \delta, \dot{\delta}) \dot{\delta} \\
\hdashline \mathrm{c}_{2}(\mathbf{q}, \dot{\mathrm{q}}) \dot{\delta}
\end{array}\right]+\left[\begin{array}{c}
\mathrm{K}_{1}(\mathrm{q}, \dot{\mathrm{q}}, \delta) \delta \\
\hdashline \mathrm{k}_{2}(\mathbf{q}, \dot{\mathrm{q}}) \delta
\end{array}\right]=\left[\begin{array}{c}
\mathbf{u} \\
\hdashline 0
\end{array}\right]
$$

where:

$\mathbf{M}$ is the inertia matrix, $q=\left(q_{1} q_{2} \ldots q_{n}\right)^{\top}$ is the vector of joint variables, $\delta=\left(\begin{array}{lllllllll}\delta_{11} & \delta_{12} & \ldots & \delta_{1 m_{1}} & \delta_{21} & \ldots & \delta_{2 m_{2}} & \ldots & \delta_{n_{n}}\end{array}\right)^{\top}$ is the vector of deflection variables,

$f_{1}$ and $f_{2}$ are the vectors containing Coriolis, centrifugal and gravitational terms, the last ones on ly in $f_{1}$,

$\mathbf{C}_{1}, \mathbf{C}_{2}, \mathrm{~K}_{1}$ and $\mathrm{K}_{2}$ are the matrices which account for the interaction of joint variables and their derivatives with deflection variables and their dérivatives; by dimensional analysis certain combinations of variables can be excluded as regards $\mathbf{C}_{2}$ and $\mathrm{K}_{2}$, which rule out any terms involving products of $\delta$ or $\dot{\delta}$ with $\delta$ or $\dot{\delta}$,

$\mathbf{u}=\left(\begin{array}{llll}\tau_{1} & \tau_{2} & \ldots & \tau_{n}\end{array}\right)^{\top}$ is the control vector of generalized forces applied at the joint variables;

in (2) appears also a nu 71 vector 0 of appropriate dimension.

Since the inertia matrix $M$ is positive definite, it can be inverted and denoted by $\boldsymbol{H}$, which can be partitioned as follows: 


$$
M^{-1}=H=\left[\begin{array}{c:c}
H_{11[n \times n]} & H_{12[n \times m ']} \\
\hdashline H_{21\left[m^{\prime} \times n\right]} & H_{22\left[m^{\prime} x^{\prime}\right]}
\end{array}\right]
$$

where $m^{\prime}=m_{1}+m_{2}+\ldots+m_{n}$. Eqs. (2) then become

$$
\begin{aligned}
& \ddot{q}=-H_{11} f_{1}-H_{12} f_{2}-H_{11} C_{1}-H_{12} C_{2}-H_{11} K_{1}-H_{12} K_{2}+H_{11} u \\
& \ddot{\delta}=-H_{21} f_{1}-H_{22} f_{2}-H_{21} C_{1}-H_{22} C_{2}-H_{21} K_{1}-H_{22} K_{2}+H_{21} u
\end{aligned}
$$

In order to put the system (4) in state space form, let

$$
\mathbf{x}=\left(\mathbf{q}^{\top} \mid \dot{q}^{\top}\right)^{\top} \quad z=\left(\delta^{\top} \mid \dot{\delta}^{\top}\right)^{\top}
$$

be the state variables; eqs. (4) become then

$$
\begin{aligned}
& \dot{x}=F x+g_{1}(x, z)+A_{1}(x, z) z+B_{1}(x, z) u \\
& \mathbf{F}=\left[\begin{array}{c|c}
0 & I \\
\hdashline 0 & 0
\end{array}\right] \quad g_{1}=\left[\begin{array}{c}
0 \\
\hdashline-H_{11} f_{1}-H_{12} f_{2}
\end{array}\right] \\
& A_{1}=\left[\begin{array}{c:c}
0 & 0 \\
\hdashline-H_{11} K_{1}-H_{12} K_{2} & -H_{11} C_{1}-H_{12} C_{2}
\end{array}\right] \quad B_{1}=\left[\begin{array}{c}
0 \\
\hdashline H_{11}
\end{array}\right] \\
& \dot{z}=A_{2}(x, z) z+g_{2}(x, z)+B_{2}(x, z) u
\end{aligned}
$$

$$
\begin{aligned}
& A_{2}=\left[\begin{array}{c:c}
0 & I \\
\hdashline-H_{21} \mathrm{~K}_{1}-\mathrm{H}_{22} \mathrm{~K}_{2} & -\mathrm{H}_{21} \mathrm{C}_{1}-\mathrm{H}_{22} \mathrm{C}_{2}
\end{array}\right] \quad \mathbf{g}_{2}=\left[\begin{array}{c}
0 \\
\hdashline-\mathrm{H}_{21} \mathrm{f}_{1}-\mathrm{H}_{22} \mathrm{f}_{2}
\end{array}\right] \\
& \mathbf{B}_{2}=\left[\begin{array}{c}
0 \\
\hdashline \mathrm{H}_{21}
\end{array}\right]
\end{aligned}
$$




\section{Singular Perturbation Formulation}

Under the assumption that the spectrum of rigid body joint motion is we 11 separated from the spectrum of flexible link deflections, the system can be considered a singularly perturbed one, at least in its open-10op configuration initial7y. In order to make this explicit, the matrix $A_{2}$ in (6b) can be written as $A_{2}^{\prime} / \mu$, where $\mu$ plays the role of the perturbation parameter. By scaling the other terms in (6b), the overall system in singularly perturbed form results

$$
\begin{aligned}
\dot{x} & =F x+g_{1}(x, z)+A_{1}(x, z) z+B_{1}(x, z) u \\
\mu \dot{z} & =A_{2}^{\prime}(x, z) z+g_{2}^{\prime}(x, z)+B_{2}^{\prime}(x, z) u
\end{aligned}
$$

As evidenced by Calise (1979), since the system (7) is nonlinear, the procedure for identifying. $\mu$ is not straightforward and may involve considerable effort, even if $f l e x i b l e$ dynamics are known to be faster than rigid dynamics. To this purpose a good estimation of $\ddot{r}$ may be given by the ratio of the highest frequency of the slow dynamics vs the smallest frequency of the fast dynamics; the range of possible joint trajectories in the manipulator workspace must be accounted for in this choice.

At this point the typical steps of singular perturbation formulation can be taken. Because of the presence of $\mu$, the system (7) exhibits a boundary Tayer phenomenon in the fast variables z. If the boundary layer decays, then the dynamics of $x$ and $z$ will vary slowly. Formally setting $\mu=0$ accomplishes an order reduction from $n+m^{\prime}$ to $n$, because the differential equations (7b) degenerate into the algebraic transcendental equations

$$
0=A_{2}(\bar{x}, \bar{z}) \bar{z}+g_{2}(\bar{x}, \bar{z})+B_{2}(\bar{x}, \bar{z}) \overline{\mathbf{u}}
$$

where the bar is used to indicate that the variables belong to the so-called 
Slow subsystem with $\mu=0$.

In order to have a system in the "standard form" (Kokotovic 1984), two crucial assumptions are to be made: a) the contributions of the deflections to the inertia matrix are negligible, i.e. $H(x, z) \cong H(x), b)$ the terms involving products of $\delta$ or $\dot{\delta}$ with $\delta$ or $\dot{\delta}$ are negligible, i.e. $\mathbf{C}_{1}(\mathbf{x}, \mathbf{z}) \cong \mathrm{c}_{1}(\mathrm{x})$, and $\mathbf{k}_{1}(\mathbf{x}, \delta) \cong \mathrm{K}_{1}(\mathbf{x})$. In this case it is possible to find the distinct ("isolated") quasi steady-state solution to (7b)

$$
\bar{z}=\left[\begin{array}{c}
{\left[\mathrm{H}_{21}(\overline{\mathbf{x}}) \mathrm{K}_{1}(\overline{\mathbf{x}})+\mathrm{H}_{22}(\overline{\mathbf{x}}) \mathrm{K}_{2}(\overline{\mathbf{x}})\right]^{-1}\left[-\mathrm{H}_{21}(\overline{\mathbf{x}}) \mathbf{f}_{1}(\overline{\mathbf{x}})-\mathrm{H}_{22}(\overline{\mathbf{x}}) \mathbf{f}_{2}(\overline{\mathbf{x}})+\mathrm{H}_{21}(\overline{\mathbf{x}}) \overline{\mathbf{u}}\right]} \\
\hdashline 0
\end{array}\right]
$$

Substituting (9) in (7a) formally yields the slow subsystem or the reduced order subsystem of (7)

$$
\begin{aligned}
& \dot{\bar{x}}= F \bar{x}+\mathbf{a}(\overline{\mathbf{x}})+B(\overline{\mathbf{x}}) \bar{u} \\
& a=g_{1}+\left[\begin{array}{c}
0 \\
\hdashline\left(H_{11} K_{1}+H_{12} K_{2}\right)\left(H_{21} K_{1}+H_{22} K_{2}\right)^{-1}\left(H_{21} f_{1}+H_{22} f_{2}\right)
\end{array}\right] \\
& B=B_{1}+\left[\begin{array}{c}
0 \\
\hdashline-\left(H_{11} K_{1}+H_{12} K_{2}\right)\left(H_{21} K_{1}+H_{22} K_{2}\right)^{-1} H_{21}
\end{array}\right]
\end{aligned}
$$

To derive the fast subsystem or the boundary 1 ayer system, it is assumed that the slow variables are constant in the boundary layer; that is $\dot{z}=0$ and $x=\bar{x}$ = constant. Operating the fast state variable change around the equilibrium trajectory $\mathbf{z}_{f}=\mathbf{z}-\overline{\mathbf{z}}$, and corresponding ly $\mathbf{u}_{f}=\mathbf{u}-\overline{\mathbf{u}}$, the fast subsystem of (7) results

$$
\mu \dot{z}_{f}=A_{z}^{\prime}(\bar{x}) z_{f}+B_{2}^{\prime}(\bar{x}) u_{f}
$$

that is 


$$
\frac{d z_{f}}{d \tau}=A_{2}^{\prime}(\bar{x}) z_{f}+B_{2}^{\prime}(\bar{x}) u_{f}
$$

where $\tau=t / \mu$ is the fast time scale.

It must be emphasized that (11) is a linear system parametrized in the slow variables $\overline{\mathbf{x}}$. Setting $\mathbf{u}_{\mathbf{f}}=\mathbf{0}$ in (11) also gives an estimate of the natural frequencies excited by the rigid body trajectory under the effect of the slow control; this point may be conveniently exploited for trajectory planning purposes.

As evidenced by the two reduced order subsystems (10) and (11), the goal now is to design the slow control $\overline{\mathbf{u}}$ which a 11 ows to track a reference joint vector trajectory, and then design the fast control $\mathbf{u}_{f}$ which stabilizes the deflections along the equilibrium trajectory which is naturaliy set by the slow system as controlled by ü.

\section{Composite control}

Under the results of the previous section, the design of a feedback control u for the ful1 system (7) can be sp 1 it into two separate designs of feedback controls $\bar{u}$ and $\mathbf{u}_{f}$ for the two reduced order systems, namely a two-time scale composite control (Chow and Kokotovic 1978)

$$
u=\bar{u}(\bar{x})+u_{f}\left(\bar{x}, z_{f}\right)
$$

with the constraint that $\mathbf{u}_{f}(\overline{\mathbf{x}}, \mathbf{0})=0$ such that $\mathbf{u}_{f}$ is inactive along the solution (9).

As far as the slow control is concerned, the reduced system (10), having as many control inputs as joint variables, shows the possibility of adopting 
well established control techniques developed for rigid manipulators, such as (Freund 1982; Luh et a 1. 1980; STotine 1985; Ba Testrino et a 1. 1983), to mention a few. In particular for the Adaptive Model Following Control technique of the type proposed in (Balestrino et a 1. 1983) the perfect model following conditions, which are the crucial point to successful use of AMFC (Landau 1979), are always satisfied, due to the structure of system (10). This issue has been addressed by Siciliano, Yuan and Book (1986) for the case of a one link flexible arm. More specifically, assume that a trajectory is assigned for the joint variables and joint velocities, say $\hat{x}(t)$, as a result of an inverse kinematic computation from the end effector trajectory for the equivalent rigid body manipulator. The slow control can be thought of as

$$
\overrightarrow{\mathbf{u}}(\overline{\mathrm{x}})=\mathrm{B}^{\dagger}(\overline{\mathrm{x}})[-\mathrm{a}(\overline{\mathrm{x}})+\mathrm{v}(\overline{\mathrm{x}}, \hat{\mathrm{x}})]
$$

where the first term provides a precompensation of the nonlinear terms in a, and $\mathbf{v}$ is a robust control which allows the slow subsystem to track a 1 inear time invariant decoupled reference mode 1 of the type

$$
\dot{\hat{\mathbf{x}}}=\left[\begin{array}{c:c}
0 & \mathrm{I} \\
\hdashline \Lambda_{0} & \Lambda_{1}
\end{array}\right] \hat{\mathbf{x}}+\left[\begin{array}{c}
0 \\
\hdashline \mathrm{I}
\end{array}\right] \hat{\mathrm{u}}
$$

with $\Lambda_{0}$ and $\Lambda_{1}$ diagonal negative matrices. It might be helpful to recal 1 from (Balestrino et a 1. 1983) that $\hat{u}$, which is strictly part of the robust control, is obtained from (14) by means of the so-called inverse model technique.

Incidentally it might be observed that the strategy of adaptively controlling the system (7a), by just dropping the flexible dynamic equations in (7b) and considering $z$ in (7a) as a disturbance to the system, is likely to fail, since no assumption on the boundedness of the disturbance can be made.

At this point the singular perturbation theory requires that the boundary layer system (11) be uniformly stable along the equilibrium trajectory $\bar{z}$ given 
in (9). This can be accomplished on ly if the couple $\left(\mathbf{A}_{2}^{\prime}, \mathbf{B}_{2}^{\prime}\right)$ in (11) is uniformly stabilizable for any slow trajectory $\bar{x}(t)$. Assuming that this holds, a fast state feedback control of the type

$$
u_{f}\left(\bar{x}, z_{f}\right)=K_{f}(\bar{x}) z_{f}
$$

wil1 stabilize the boundary layer system (11) to $\mathbf{z}_{f}=\mathbf{0}(\mathbf{z}=\overline{\mathbf{z}})$. According to Chow and Kokotovic (1978), $\mathbf{u}_{f}$ can be designed as on optimal control for the boundary layer. The performance index will be a function of the slow state variables. Since the main purpose in flexible manipulator control is to damp out the deflections at steady-state as fast as possible, $k_{f}$ can be designed a.1so on the basis of the final joint configuration, provided that with that particular choice $z_{f}$ will not go unstable along the slow trajectory; in this way the solution of a Riccati equation for each joint configuration can be avoided.

A crucial issue for the composite control synthesis is that the slow control (13) and the fast control (15) must be designed so as to preserve the time scale separation for the closed-loop system.

Under the above conditions Tikhonov's theorem (Kokotovic 1984) assures that the state vectors can be approximated by

$$
\begin{aligned}
& x=\bar{x}+\mathbf{0}(\mu) \\
& z=\bar{z}+z_{f}+0(\mu)
\end{aligned}
$$

Under the slow control (13), $\bar{x}$ will tend to $\hat{x}$. The fast control (15) will drive $z_{f}$ to 0 . The goal of tracking the joint trajectory and stabilizing the deflections around the quasi steady-state trajectory, naturally set up by the slow system under the slow control, is then achieved by an $\mathbf{0}(\mu)$ approximation. This is the typical result of a singular perturbation approach. 


\section{Case study}

The control strategy outlined in the above sections has been tested for the one 1 ink flexible arm existing in the Flexible Automation Laboratory at Georgia Tech; the arm is sketched in fig. 1. For reader's convenience the whole dynamic model with two mode expansion (1) in the form (2) is reported in Appendix. Under the assumption of neglecting the contributions of the deflections to the inertia matrix and the second order terms in the deflection variables and their derivatives as previously anticipated, it is $\boldsymbol{M}=$ constant in (A2) and $c_{1}{ }^{\top}=\left(\begin{array}{ll}0 & 0\end{array}\right)$ in (A5) respectively. This system verifies the basic assumption required for the time scale separation, since the open loop spectra are we 11 separated; the rigid body motion involves two poles at the origin (no gravity), and the natural frequencies associated with the flexible motion maintain around $14 \mathrm{rad} / \mathrm{s}$ and $90 \mathrm{rad} / \mathrm{s}$ along the whole trajectory.

Assume to indicate the desired trajectory which defines the reference

mode 1 in (13) by $(\hat{\theta}, \dot{\hat{\theta}}, \ddot{\hat{\theta}})$. A joint trajectory is commanded from $\hat{\theta}(0)=0^{\circ}$ to $\hat{\theta}(T)=90^{\circ}$ and $T=2 \mathrm{~s}$, with the velocity profile $\dot{\hat{\theta}}=90[1+\sin (2 \pi t / T-\pi / 2)]$ [\%/s] (fig. 2)

Since the one link case does not involve high nonlinearities, the slow control in (13) has been chosen as a linear model following control

$$
\overline{\mathrm{u}}=\frac{1}{\mathrm{~h}_{11}}\left[\ddot{\hat{\theta}}+\mathrm{k}_{\mathrm{v}}(\dot{\hat{\theta}}-\dot{\bar{\theta}})+\mathrm{k}_{\mathrm{p}}(\hat{\theta}-\bar{\theta})\right]
$$

The choice for $k_{p}$ and $k_{v}$ in (17) is to be made on the basis of a tradeoff between high tracking accuracy and validation of the two time scale separation for the closed 10op system. In other words if the natural frequency associated with $k_{p}$ and $k_{v}$ is too high, the closed-loop poles will not be clustered anymore into slow and fast poles matching the open-loop time scale separation 
(Khalil 1985). Since the smaller natural frequency of the fast subsystem is around $14 \mathrm{rad} / \mathrm{sec}$, according to a ratio of 1 to 6 for the perturbation parameter, a double pole in -2.5 has been chosen for the reference model, corresponding to $k_{p}=6.25$ and $k_{v}=5$.

The fast control in (15) can be selected according to optimal control with a prescribed degree of stability, whose performance index is

$$
J=\int_{0}^{\infty}[\exp (-\alpha \tau)]\left[\mathbf{z}_{f}^{\top} \mathbf{Q}(\overline{\mathbf{x}}) \mathbf{z}_{f}+r(\overline{\mathbf{x}}) \mathrm{u}_{f}^{2}\right] \mathrm{d} \boldsymbol{\tau}
$$

The resulting fast optimal control is $\left(\mathbf{b}_{2}^{\prime}\right.$ in (11) is a constant vector)

$$
u_{f}\left(\bar{x}, z_{f}\right)=-\frac{1}{r(\bar{x})} b_{2}^{\prime T} v(\bar{x}) z_{f}
$$

where $\mathbf{V}(\overline{\mathbf{x}})$ is the positive definite stabilizing solution of the $\overline{\mathbf{x}}$-dependent Riccati equation

$$
0=-V(\overline{\mathbf{x}})\left[A_{2}^{\prime}(\overline{\mathbf{x}})+a I\right]-\left[A_{2}^{\prime \top}(\overline{\mathbf{x}})+a I\right] V(\overline{\mathbf{x}})+V(\overline{\mathbf{x}}) \mathrm{b}_{2}^{\prime} b_{2}^{\prime \top} V(\overline{\mathbf{x}}) / r(\overline{\mathbf{x}})-Q(\overline{\mathbf{x}})
$$

In order to achieve good damping at steady-state and a 7 so save computation time, the fast optimal control has been designed for the final joint configuration $\overline{\mathbf{x}}^{\top}=\left[\begin{array}{ll}90 & 0\end{array}\right]$. The penalty terms in (18) have been chosen as $\mathbf{Q}=$ diag( 115030000$)$ and $r=1$ respective1y, also $a=5$. With these values the damping ratios for the two fast modes have resulted .766 and .753 respectively, and the natural frequencies 18.59 and 94.10 respectively. This last point actually meets the constraint of preserving the time scale separation existing for the open-loop system. The resulting $k_{f}^{T}$ is $\left(\begin{array}{llll}75.50 & 6375.64 & 17.07 & 173.10\end{array}\right)$.

In order to test the robustness of the composite control, the whole non linear system given in Appendix has been simulated. The pure slow control 
has been first applied, then the fast control has been added. Fig. 3 displays the joint angle tracking performance, and fig. 4 the joint velocity tracking performance. Figs. 5 and 6 show the two modal deflections, while figs. 7 and 8 show the derivatives of the modal deflections. Furthermore the end point tracking performance, position and velocity respectively, are reported in figs. 9 and 10. As could have been predicted, the joint angle tracking proves even better under the effect of the pure slow control, but at steady-state both the joint angle and the deflections, are not stabilized at a 11 and they seem to damp very slowly. This effect is also visible for the end point tracking. The addition of the fast stabilizing control contributes to stabilize the deflections along the trajectory and damp them out at steadystate, while not derating appreciably the tracking performance. This point reflects the compromise which must be achieved in designing a control system for a flexible link arm. Fig. 11 finally shows the required net input torques, the slow, the fast and the composite respectively. It might be remarked that the shapes of the controls are gentle and their magnitudes contained.

\section{Concluding remarks}

A singular perturbation approach has been developed for control of 1 ightweight flexible manipulators moving along predefined paths.

The main problem concerned with flexible arm tracking control, namely the number of control inputs is less than the number of controlled variables, has been successfulty faced by means of a model order reduction which is characteristic of a two-time scale approach. Indeed for the slow subsystem we 17 established control strategies, as for rigid arms, can be adopted. For the fast subsystem a stabilizing control along the quasi steady-state 
trajectory, set up by the slow subsystem as controlled by the slow control, is needed. The control goal is achieved by an $\mathbf{0}(\mu)$ approximation, where $\mu$ represents the ratio of the speeds of the slow vs the fast dynamics. However, since $\mu$ is not identified in a rigorous manner, the approach presented is recommendable only if it leads to useful and accurate solutions, i.e. $\mu$ is sufficient $7 y$ sma 11. To this end the time scale separation must be preserved in designing the two feedback controls. The stability of the full order system, indeed, has not been direct ly addressed in this paper. Estimates of the domain of attraction and of an upper bound on the perturbation parameter are still being sought, and they constitute the main focus of the current research.

Future research effort will be also likely dedicated to seek integral manifolds for the slow subsystem (Sobolev 1984), in order to obtain a more accurate slow control, especially for the case of multi-degree-of-freedom flexible manipulators.

Long term research issues are:

- how to select "good" joint angle trajectories so as not to excite large deflections and then lighten the task of the fast control,

- to better investigate the effect of the slow control on the natural frequencies of the fast dynamics.

Ultimately the problem of full state reconstruction and the possibility of adopting output feedback, i.e. dropping the derivatives of deflection variables and eventual1y using a dynamic compensator, certainly represent two challenging points to investigate to a greater extent. 


\section{Acknow ledgements}

Bruno Siciliano wishes to acknow ledge the support of this work by the NAT0 Science Programme (Special Programme on Sensory Systems for Robotic Control) and the Computer Integrated Manufacturing Systems Program at the Georgia Institute of Technology where the author was on leave as Visiting Scholar. The encouragement of Lorenzo Sciavicco and Giuseppe De Maria was also important.

\section{REFERENCES}

Balestrino, A., De Maria, G., and Sciavicco, L. 1983. An adaptive model following control for robotic manipulators. Trans. ASME J. Dyn. Syst. Measurement Contr., vol. 105, no. 3, pp. 143-151.

Book, W.J. 1984 (August). New concepts in 1 ightweight arms. Proc. 2nd Int. Symp. Robotics Research, Kyoto, Japan.

Book, W.J. 1984. Recursive Lagrangian dynamics of flexible manipulator arms. Int. J. Robotics Research, vo 1. 3, no. 3, pp. 87-101.

Book, W.J., Le, S., and Sangveraphunsiri, V. 1984 (June). The bracing strategy for robot operation. Proc. 5th CISM-IFTOMM Symp. Theory Robots Manipulators (Ro.Man.Sy.), Udine, I.taTy.

Calise, A.J. 1979. A singular perturbation analysis of optimal aerodynamic and thrust magnitude contro1. IEEE Trans. Automat. Contr., vol. AC-24, no. 5, pp. $720-730$.

Chow, J.H., and Kokotovic, P.V. 1978. Two-time-scale feedback design of a class of nonlinear systems. IEEE Trans. Automat. Contr., vol. AC-23, no. 3, pp. $438-443$.

Freund, E. 1982. Fast nonlinear control with arbitrary pole placement for industrial robots and manipulators. Int. J. Robotics Research, vol. 1, no. 1 , pp. 65-78.

Hastings, G.G., and Book, W.J. 1985 (June). Experiments in optimal control of a flexible arm. Proc. 1985 American Contr. Conf., Boston, MA.

Hastings, G.G., and Book, W.J. 1986 (Apr.). Verification of a 1 inear dynamic model for flexible manipulators. Proc. 1986 Int. Conf. Robotics Automation, San Francisco, CA.

Khali T, H.K. 1985 (June). Output feedback of linear two time scale systems. Proc. 1985 American Contr. Conf., Boston, MA. 
Kokotovic, P.V. 1984. Applications of singu lar perturbation techniques to control problems. SIAM Review, vol. 26, no. 4, pp. 501-550.

Landau, Y.D. 1979. Adaptive Contro 1: The Mode 1 Reference Approach, New York, NY, Marcel Dekker, Inc.

Luh, J.Y.S., Wa 1ker M.W., and Pau 7 R.P.C. 1980. Reso 1 ved acceleration control of mechanical manipulators. IEEE Trans. Automat. Contr., vol. AC-25, no. 3, pp. $468-474$.

Maizza Neto 0. 1974. Modal analys is and control of flexible manipulator arms. Ph.D. thesis. Massachusetts Institute of TechnoTogy, Department of Mechanical Engineering.

Marino, R., and Nicosia, S. 1985 (Nov.). Singular perturbation techniques in the adaptive control of elastic robots. Proc. IFAC Symp. Robot Control (Sy.Ro.Co.), Barcelona, Spain.

Me 1drum, D.R. 1985. Direct mode 1. reference adaptive control of a flexible robotic manipulator. M.S. thesis. Rensselaer Polytechnic Institute.

Sangveraphunsiri, V. 1984. The optimal control and design of a flexible robotic manipulator. Ph.D. thesis. Georgia Institute of Technology, School of Mechanical Engineering.

Siciliano, B., Yuan, B.-S., and Book, W.J. 1986 (Dec.). Mode 1 reference adaptive control of a one link flexible arm. 25 th IEEE Conf. Decision Contr., Athens, Greece, submitted for presentation, aTso IEEE Trans. Automat. Contr., submitted for publication.

Singh, S.N., and Schy, A.A. 1985 (June). Decomposition and state variable feedback control of elastic robotic systems. Proc. 1985 American Contr. Conf., Boston, MA.

Slotine, J.-J.E. 1985. The robust control of robot manipulators. Int. J. Robotics Research, vol. 4, no. 2, pp. 49-64.

Sobolev, V.A. 1984. Integral manifolds and decomposition of singularly perturbed systems. Systems Contr. Letters, vo1. 5, pp. 1169-1179.

Suzuki, M. 1981. Composite controls for singularly perturbed systems. IEEE Trans. Automat. Contr., vol. AC-26, no. 2, pp. 505-507. 


\section{Appendix}

The dynamic model of the one link flexible arm is presented. The modal series $\overline{(1)}$ is truncated at $m_{1}=2$. For a clamped-free vibrating beam the orthonormal modal eigenfunctions in (1) are given by

$$
\begin{gathered}
\phi_{i}(\xi)=\sin \left(\beta_{i} \xi\right)-\sinh \left(\beta_{i} \xi\right)+\nu_{i}\left[\cos \left(\beta_{i} \xi\right)-\cosh \left(\beta_{j} \xi\right)\right] \\
\nu_{i}=\frac{\sin \beta_{i}+\sinh \beta_{i}}{\cos \beta_{i}+\cosh \beta_{i}}, \quad \beta_{i}^{4}=\frac{\rho A\left(2 \pi f_{i}\right)^{2} L^{4}}{E I} \quad i=1,2 \\
\xi=\eta / L
\end{gathered}
$$

With reference to the Lagrangian dynamic equations in the form (2), it results:

$$
\begin{aligned}
& \mathbf{M}=\left[\begin{array}{lll}
m_{11} & m_{12} & m_{13} \\
m_{12} & m_{22} & m_{23} \\
m_{13} & m_{23} & m_{33}
\end{array}\right] \\
& m_{11}=J_{0}+M L^{2}+I_{0}+M\left(\phi_{1 e}^{2} \delta_{1}^{2}+2 \phi_{1 e} \phi_{2 e} \delta_{1} \delta_{2}+\phi_{2 e}^{2} \delta_{2}^{2}\right) \\
& \quad m_{12}=M L \phi_{1 e}+w_{1} \\
& m_{13}=M L \phi_{2 e}+w_{2} \\
& m_{22}=m+M \phi_{1 e}^{2}+J_{p} \phi_{1 e}^{\prime 2} \\
& m_{23}=M \phi_{1 e} \phi_{2 e}+J_{p} \phi_{1 e}^{\prime} \phi_{2 e}^{\prime} \\
& m_{33}=m+M \phi_{2 e}^{2}+J_{p} \phi_{2 e}^{\prime 2} \\
& f_{1}=0 \\
& \mathbf{f}_{2}=\left(\begin{array}{ll}
0 & 0
\end{array}\right)^{\top} \\
& c_{1}^{\top}=2 M\left[\left(\phi_{1}^{2} \delta_{1}+\phi_{1 e} \phi_{2 e} \delta_{2}\right) \quad\left(\phi_{1 e} \phi_{2 e \delta_{1}}+\phi_{2}^{2} \delta_{2}\right)\right]
\end{aligned}
$$


?

$$
\begin{aligned}
& c_{2}=\left[\begin{array}{ll}
0 & 0 \\
0 & 0
\end{array}\right] \\
& k_{1}^{\top}=\left(\begin{array}{ll}
0 & 0
\end{array}\right) \\
& k_{2}=\left[\begin{array}{l}
k_{11} k_{12} \\
k_{12} k_{22}
\end{array}\right] \\
& k_{11}=k_{e 1}-M \dot{\theta}^{2} \phi_{1 e} \\
& k_{12}=-M \dot{\theta}^{2} \phi_{1} \phi_{2 e} \\
& k_{22}=k_{e 2}-M \dot{\theta}^{2} \phi_{2 e}
\end{aligned}
$$

where:

$$
\begin{aligned}
& \phi_{i e}=\left.\phi_{i}(\xi)\right|_{\xi=1} \quad i=1,2 \\
& \phi_{i e}^{\prime}=\left.\frac{d \phi_{i}(\xi)}{d \xi}\right|_{\xi=1} \quad i=1,2 \\
& w_{i}=\rho A L^{2} \int_{0}^{1} \phi_{j}(\xi) \xi d \xi \quad i=1,2 \\
& k_{e i}=\frac{E I}{L^{3}} \int_{0}^{1}\left[\frac{d^{2} \phi_{j}(\xi)}{d \xi^{2}}\right]^{2} d \xi \quad i=1,2 .
\end{aligned}
$$

Futhermore the end point position $\chi_{e}$ and velocity $\dot{\chi}_{e}$ along the $x_{0}$ axis result respectively:

$$
\begin{aligned}
& x_{\mathrm{e}}=\left.x\right|_{\xi=1}=L \cos \theta-\left(\phi_{1 \mathrm{e}} \delta_{1}+\phi_{2 \mathrm{e}} \delta_{2}\right) \sin \theta \\
& \dot{\chi}_{\mathrm{e}}=\left.\dot{x}\right|_{\xi=1}=\left[-L \sin \theta-\left(\phi_{1 \mathrm{e}} \delta_{1}+\phi_{2 \mathrm{e}} \delta_{2}\right) \cos \theta\right] \dot{\theta}-\left(\phi_{1 \mathrm{e}} \dot{\delta}_{1}+\phi_{2 \mathrm{e}} \dot{\delta}_{2}\right) \sin \theta
\end{aligned}
$$

20 
Table of simbols

$L=$ beam 1 engt $h$

$\mathrm{m}=$ beam mass

$M=$ pay load mass

$I_{0}=$ joint inertia

$J_{0}=$ beam inertia relative to joint

$J_{p}=$ pay load inertia

$A=$ beam cross area

$E=$ Young's modu 1us

$I$ = beam area inertia

$\rho=$ density

$f_{i}=$ frequency of the ith mode

For the prototype in the laboratory the above parameters are found to be (Hastings and Book 1985):

$L=4 \mathrm{ft}$

$m=0.02051 b_{f} s^{2} / f t$

$M \stackrel{!}{=} 3.10 \mathrm{E}-3 \quad 1 \mathrm{~b}_{\mathrm{f}} \mathrm{s}^{2} / \mathrm{ft}$

$I_{0}=1.007 b_{f} s^{2} / f t$

$\mathrm{J}_{0}=0.1091 \mathrm{~b}_{\mathrm{f}} \mathrm{s}^{2} / \mathrm{ft}$

$J_{p}=8.52 \mathrm{E}-3 \quad 1 \mathrm{~b}_{\mathrm{f}} \mathrm{s}^{2} / \mathrm{ft}$

$A=9.76 E-4 f t^{2}$

$E I=28.61 b_{f} f t^{2}$

$\rho=5.251 b_{f} s^{2} / f t^{4}$

$f_{1}=2.12 \mathrm{~Hz}$

$f_{2}=14.3 \mathrm{~Hz}$ 
FIGURE CAPTIONS

Fig. 1. The one link flexible arm.

Fig. 2. The reference joint velocity profile.

Fig. 3. Joint angle tracking errors.

Fig. 4. Joint velocity tracking errors.

Fig. 5. 1st mode deflections.

Fig. 6. 2nd mode deflections.

Fig. 7. 1st mode deflection derivatives.

Fig. 8. 2nd mode deflection derivatives.

Fig. 9. End point position tracking errors.

Fig. 10. End point velocity tracking errors.

Fig. 11. Control torques. 


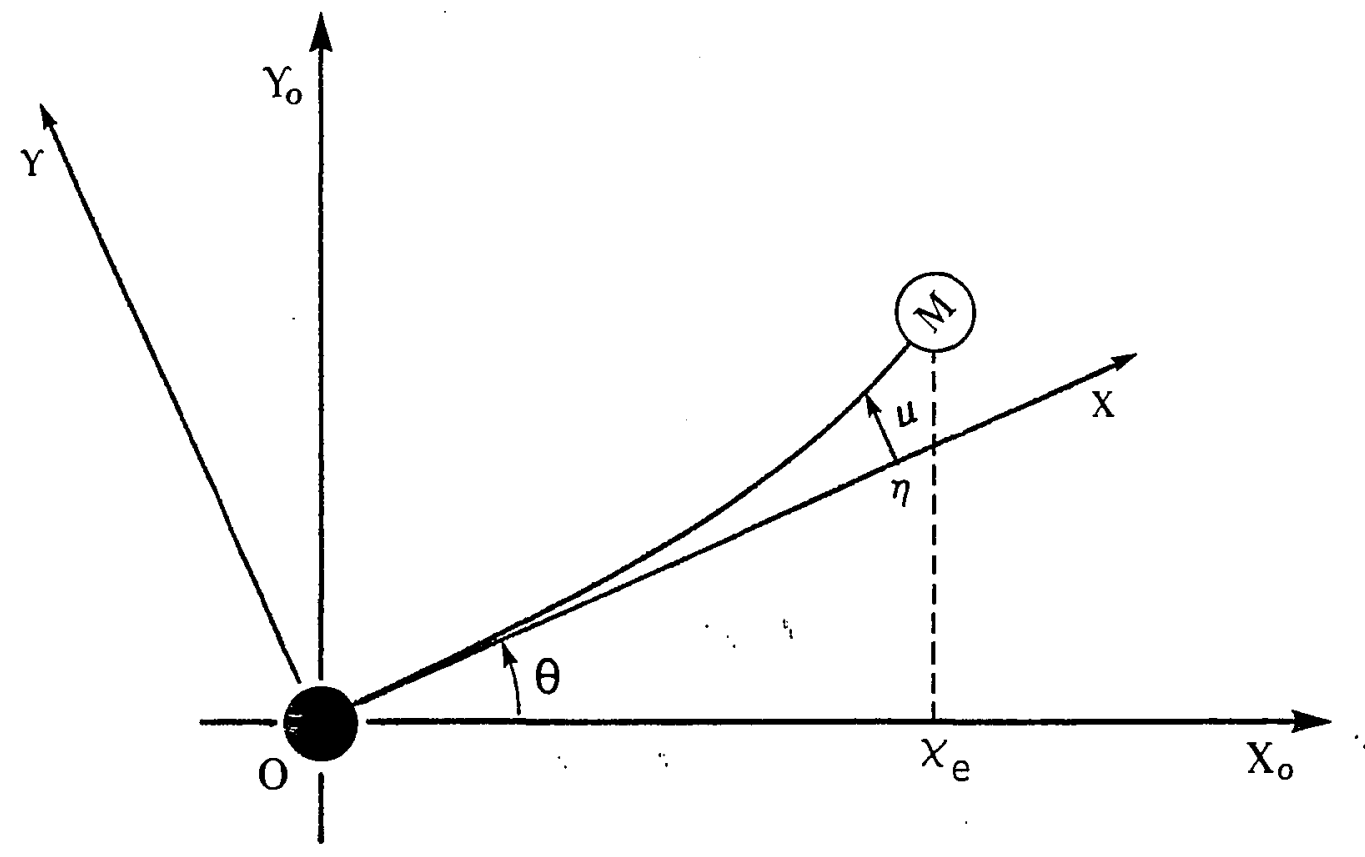

Fig. 1 


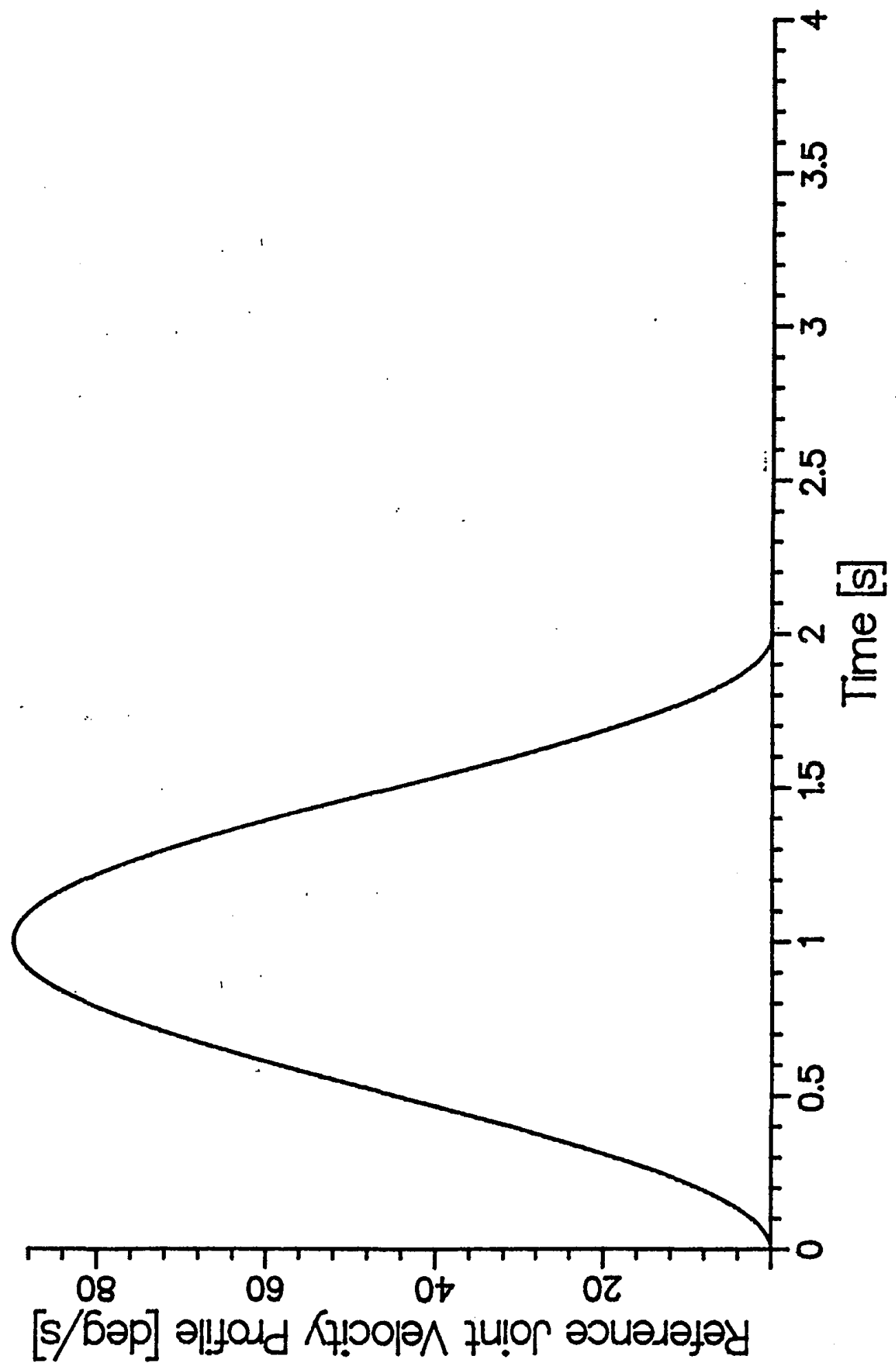




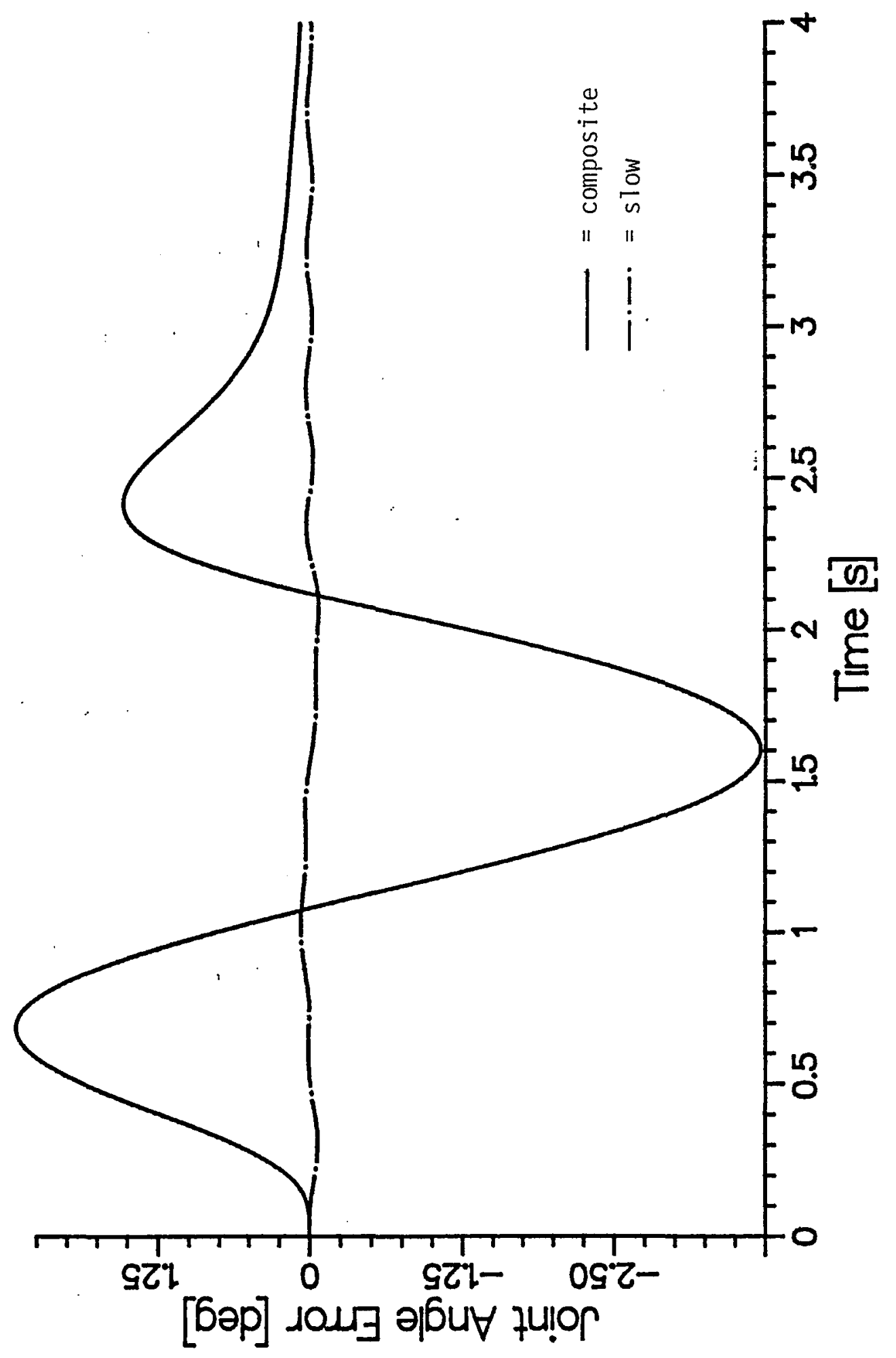




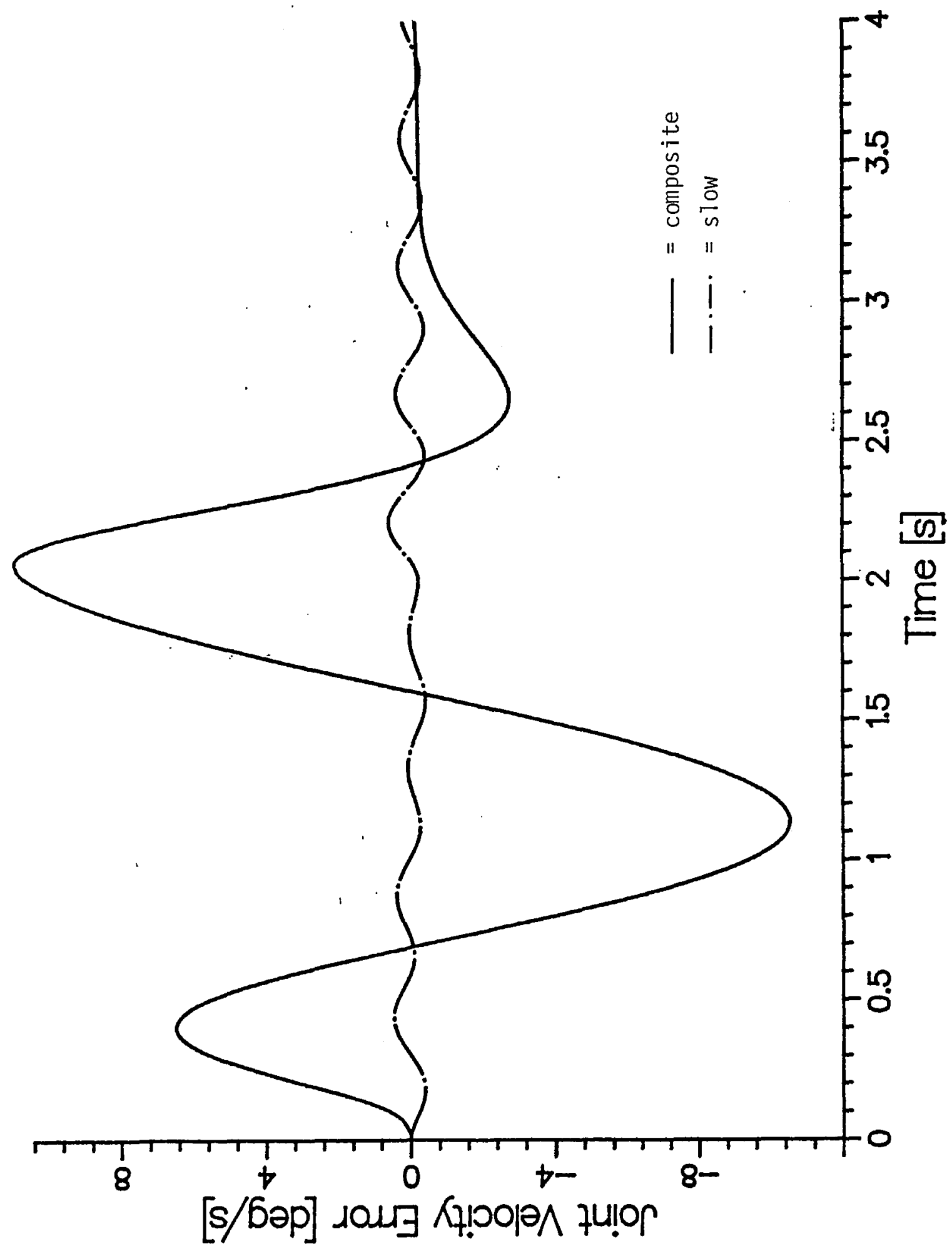




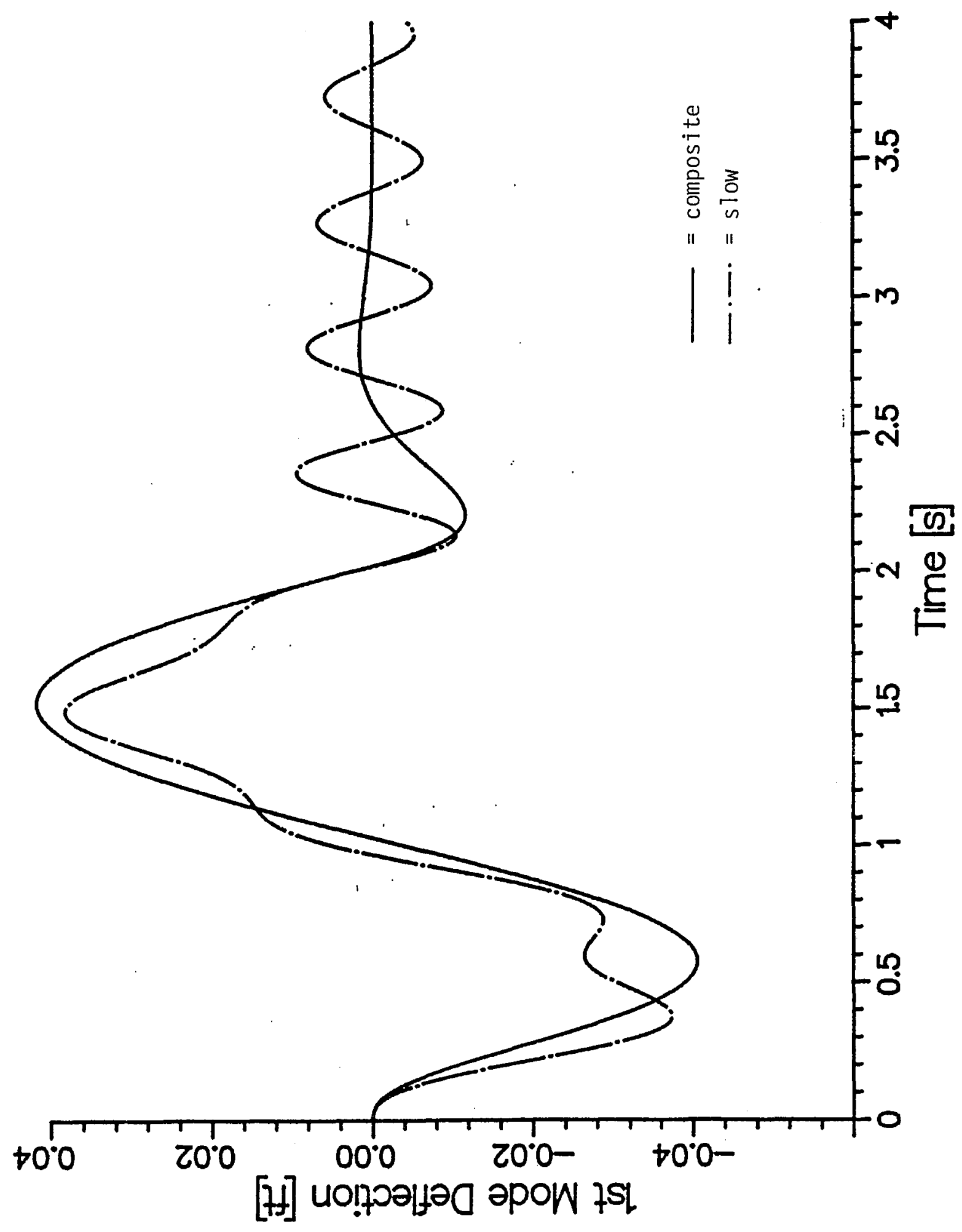




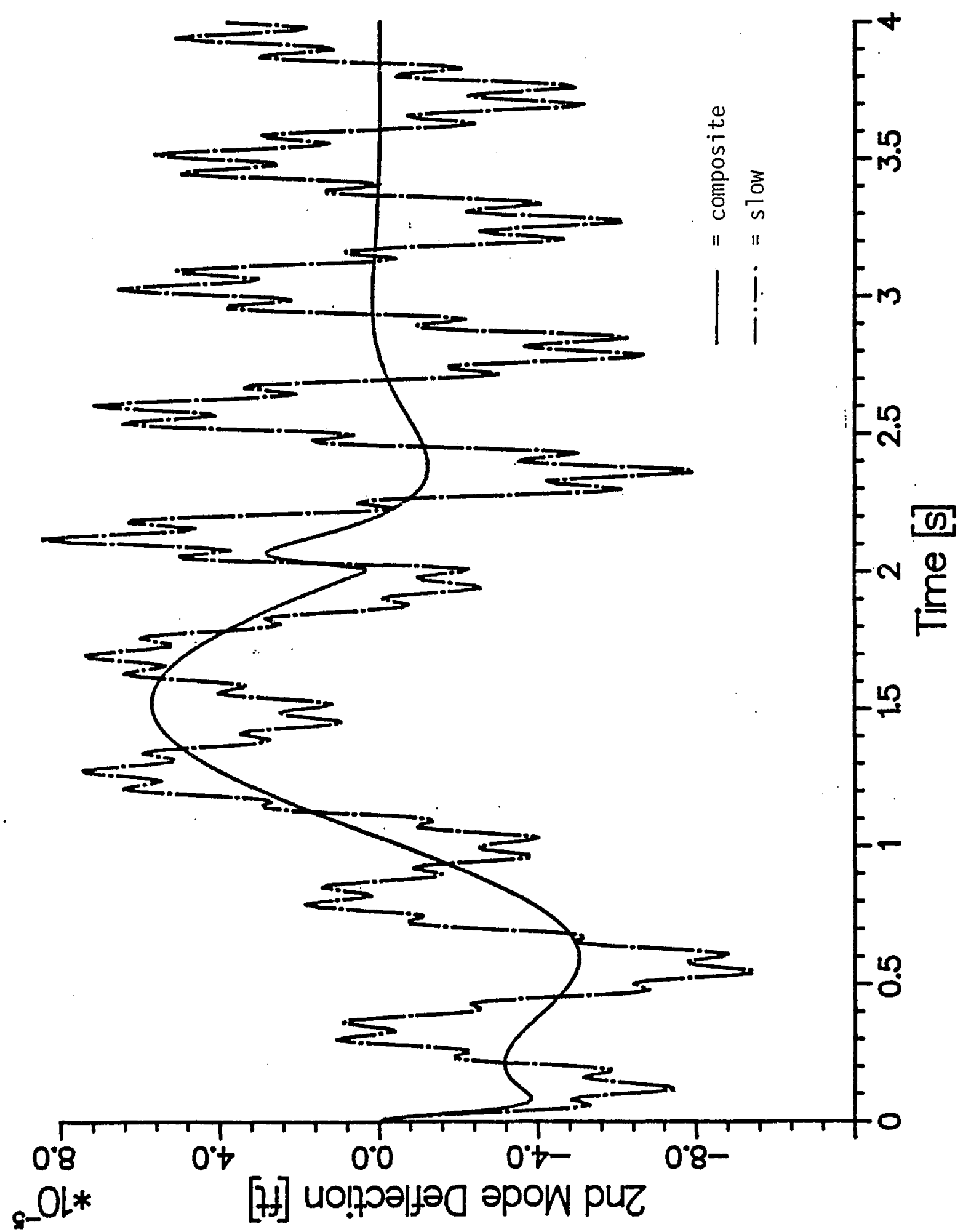




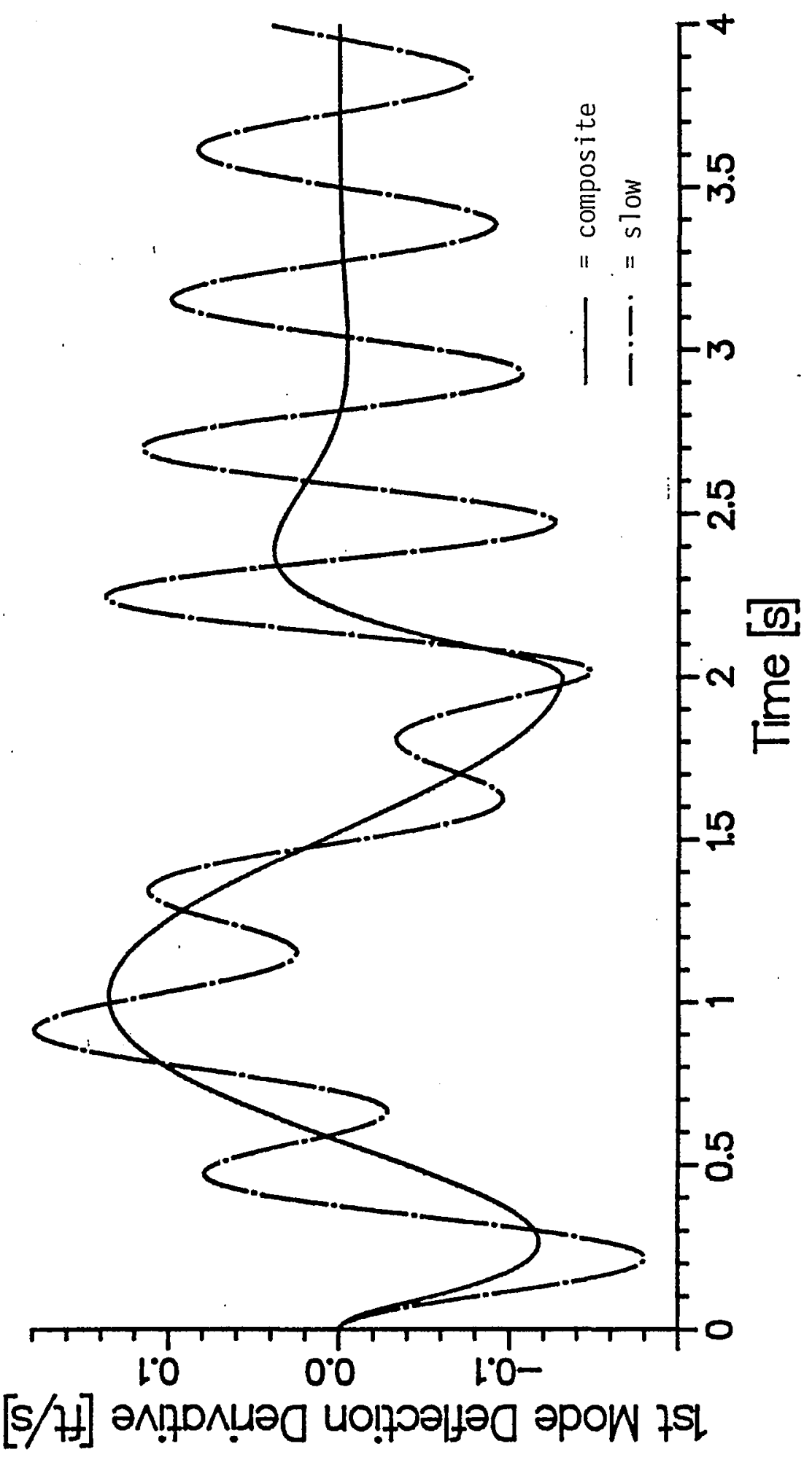




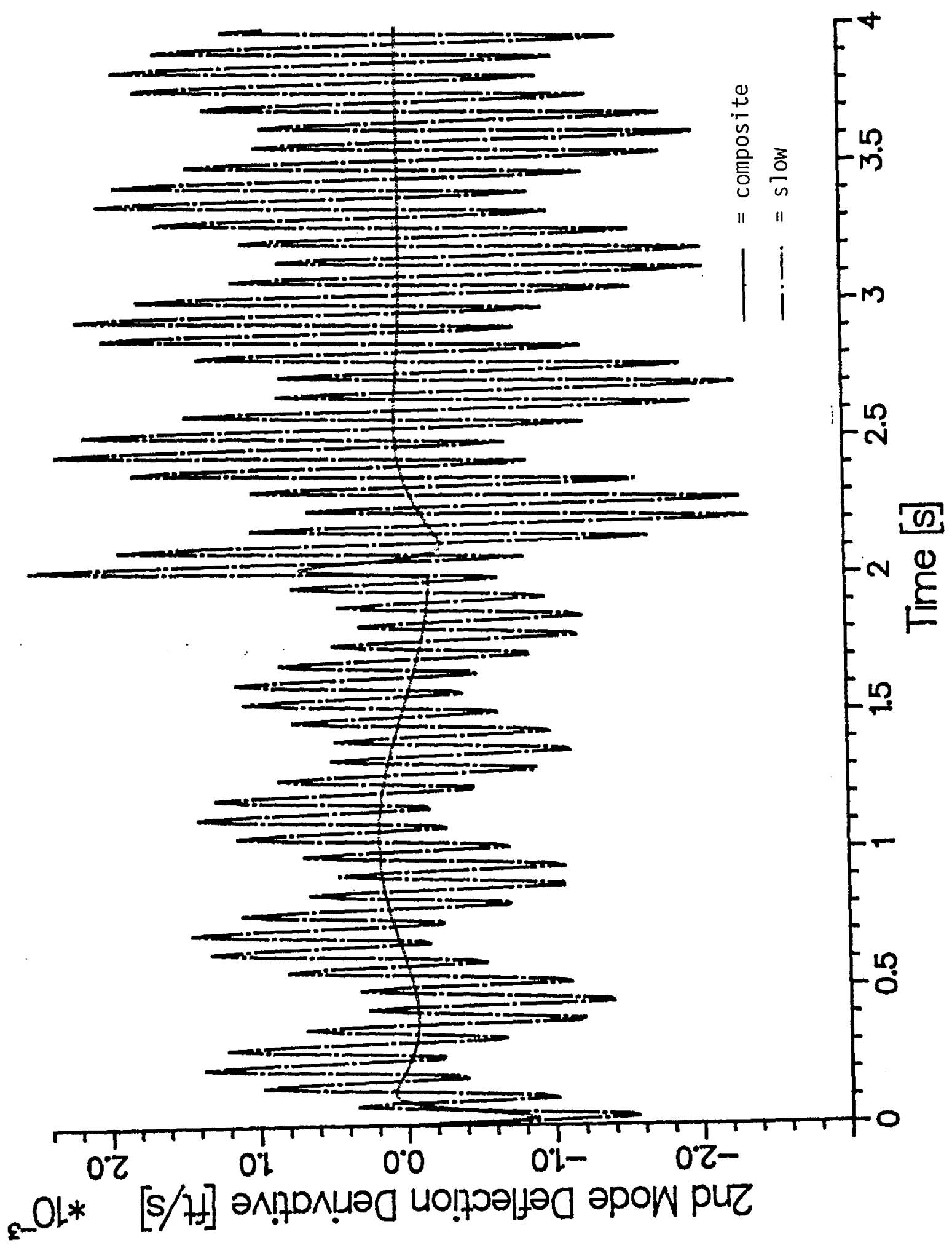




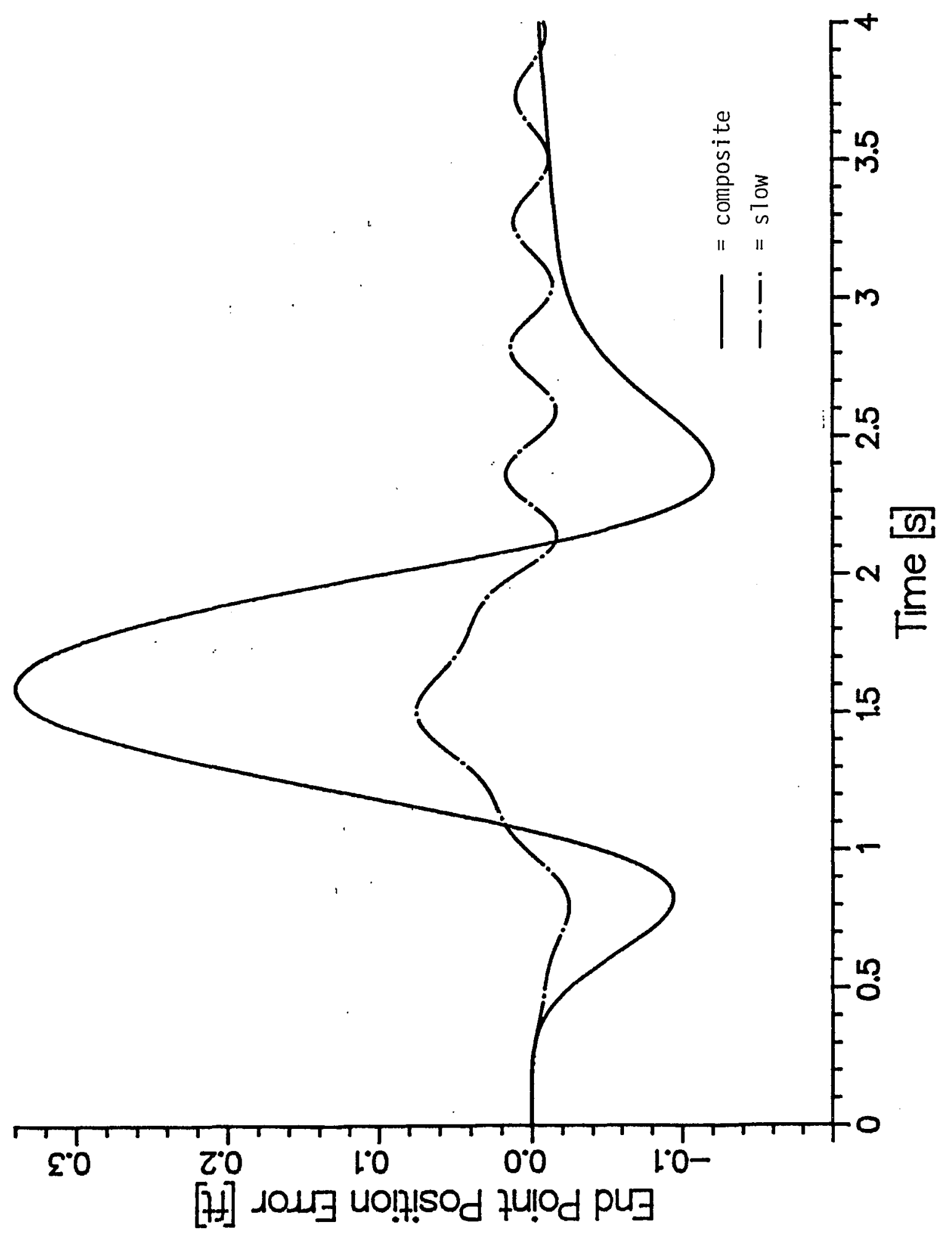




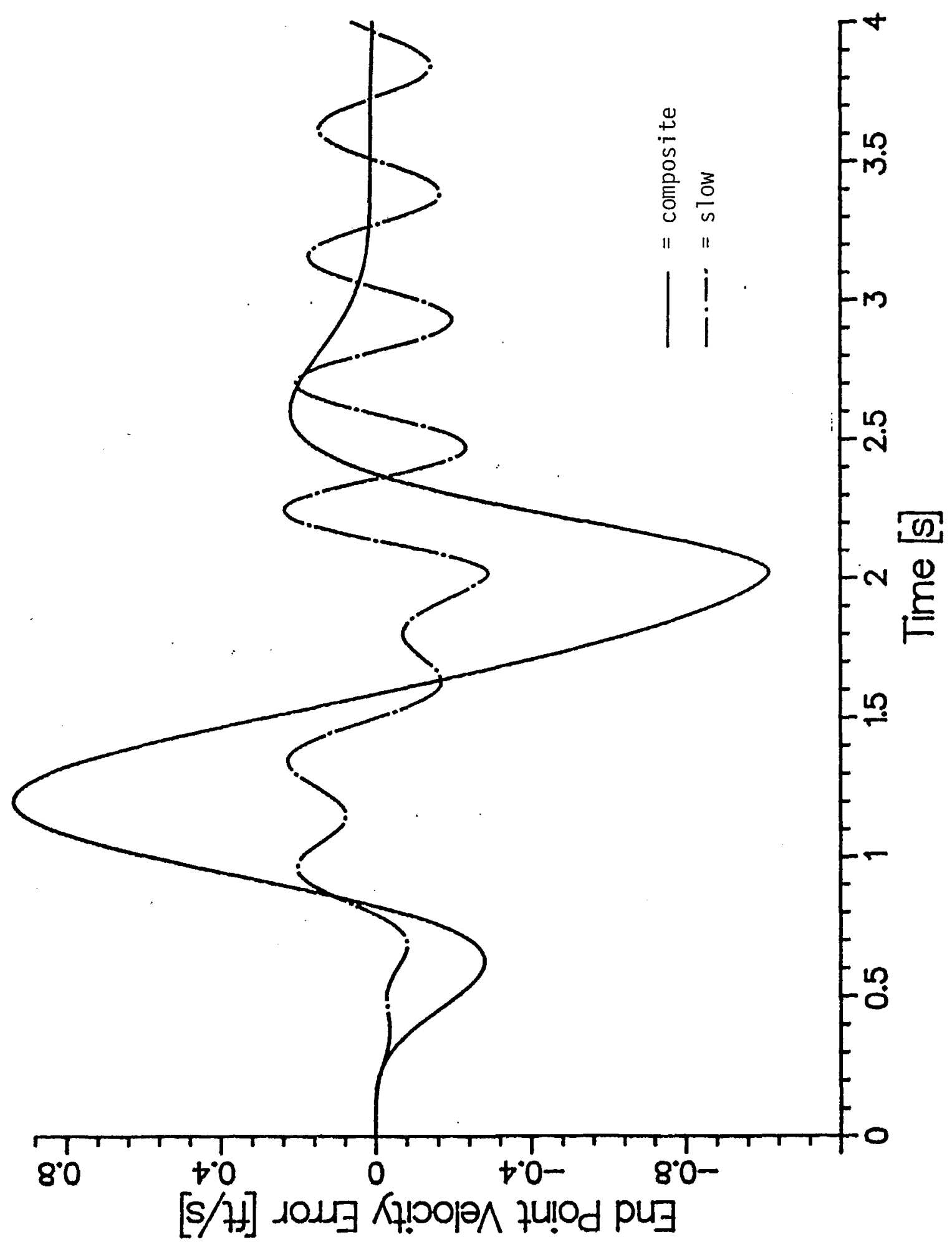




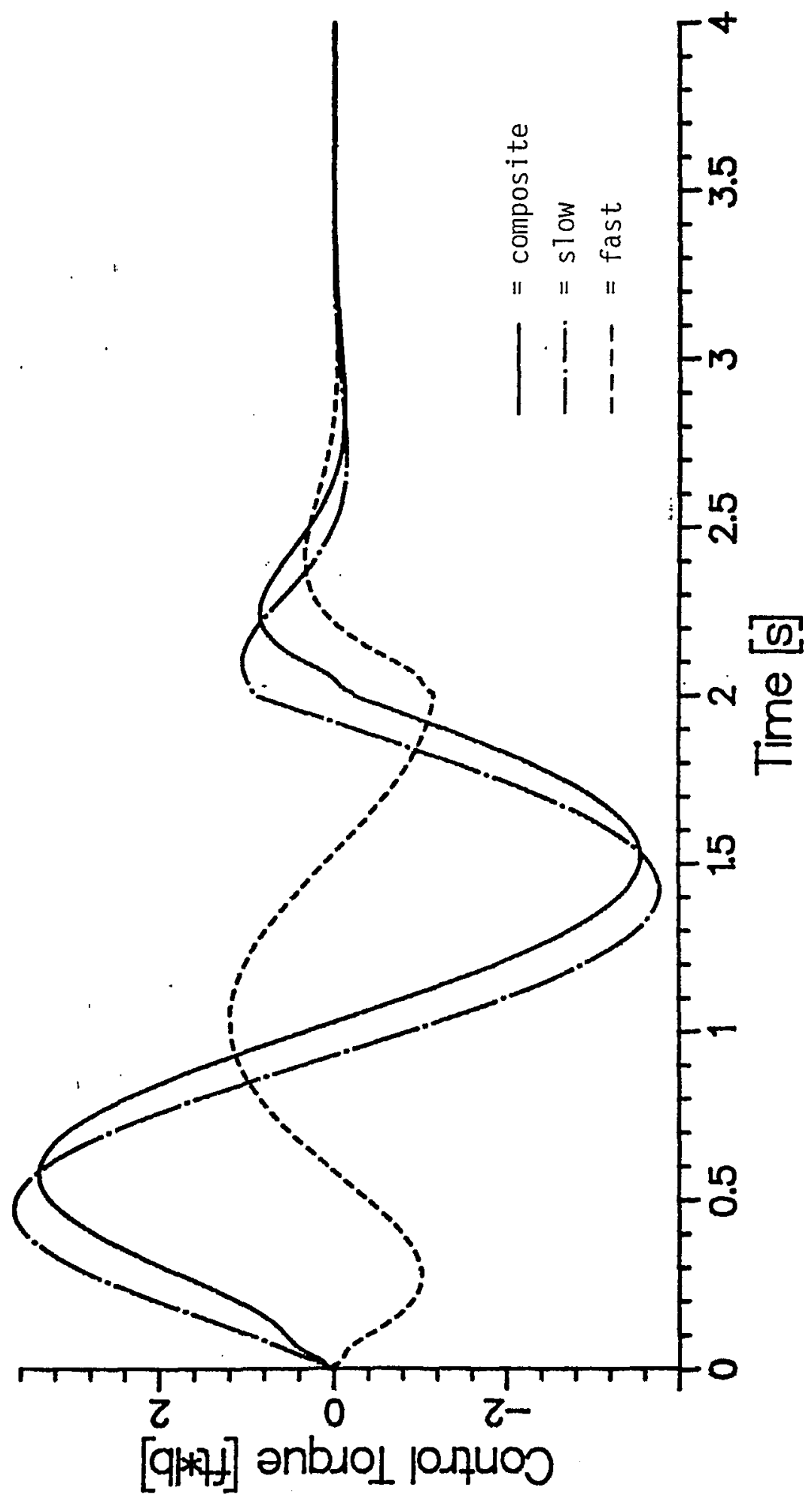

